\title{
Active Multi Degree-of-Freedom Pendulum Tuned Mass Damper
}

\author{
M. Eltaeb ${ }^{1}$, R. Kashani ${ }^{2}$ \\ ${ }^{1}$ Misurata University \\ Misurata, Libya \\ ${ }^{2}$ University of Dayton \\ Dayton, OH, USA \\ reza.kashani@udayton.edu
}

\begin{abstract}
A novel active pendulum tuned mass damper (APTMD) configuration along with a multi-layer control strategy for mitigating the vibration of tall structures, are presented. The control scheme a) increases the damping effectiveness of the APTMD and b) enables the APTMD to simultaneously tune itself to multiple frequencies adding tuned damping to the corresponding modes of those frequencies. The enhanced damping effectiveness component of the proposed control scheme and the multi-frequency tuning attribute of the proposed control strategy, eliminating the need for using multiple TMDs tuned to different frequencies, result in the control of structural vibration using a relatively small mass. The superior damping effectiveness and multi-frequency tuning capacity of the proposed APTMD are numerically demonstrated by incorporating it into the model of a multi-degree of freedom asymmetrical tall building. The APTMD introduced as much damping to the target mode as a passive PTMD twice as large, while tuned to three modes of the building.
\end{abstract}

Keywords: Active Tuned Mass Damper, Vibration Control, High-Rise Building, Multi-Frequency Tuning.

\section{Introduction}

Tuned mass dampers have been extensively used for adding tuned damping to various structures. Considering that the first vibrational mode of most structures plays the dominant role in their dynamic response, TMDs are normally tuned (and must remain tuned) to the first natural frequency of these structures. The damping effectiveness of a TMD depends on a) the size of its mass compared to the modal mass of its target mode, i.e., its mass ratio, b) its internal damping, and c) the accuracy of its tuning.

Due to the tight restriction on their weight and space requirement, the mass ratio of a TMD used in a large structure such as a tall building, is very small. The small mass ratio necessitates the use of low internal damping in the TMD, itself. The highly underdamped nature of such TMD results in

o a very narrow operating frequency range (bandwidth) making the effectiveness of the TMD highly sensitive to its tuning accuracy (i.e., a slight change in the dynamics of the target structure would render such TMD detuned and hence less effective),

o a rather long delay in reaching steady state motion (and thus becoming fully effective) when a disturbance starts perturbing the structure and also a long delay to stop when the disturbance has ceased,

$o$ excessive excursion, and

o low damping effectiveness even when the TMD with small mass ratio is well-tuned.

The impact of mass ratio on the damping effectiveness of a TMD is evident from Figure 1 depicting the frequency response functions of a structure without and with two different TMDs, one with a small $(0.125 \%)$ and the other with a large $(0.5 \%)$ mass ratio. As enumerated above and also clear from Figure 1, the TMD with smaller mass ratio has 1) lower bandwidth, and 2) less damping effectiveness than the TMD with larger mass ratio.

The above-listed issues could have been addressed if the mass ratio of the TMD was large. But a large mass ratio would make the TMD considered for a high-rise building very massive, excessively large, and unacceptable in most applications. 


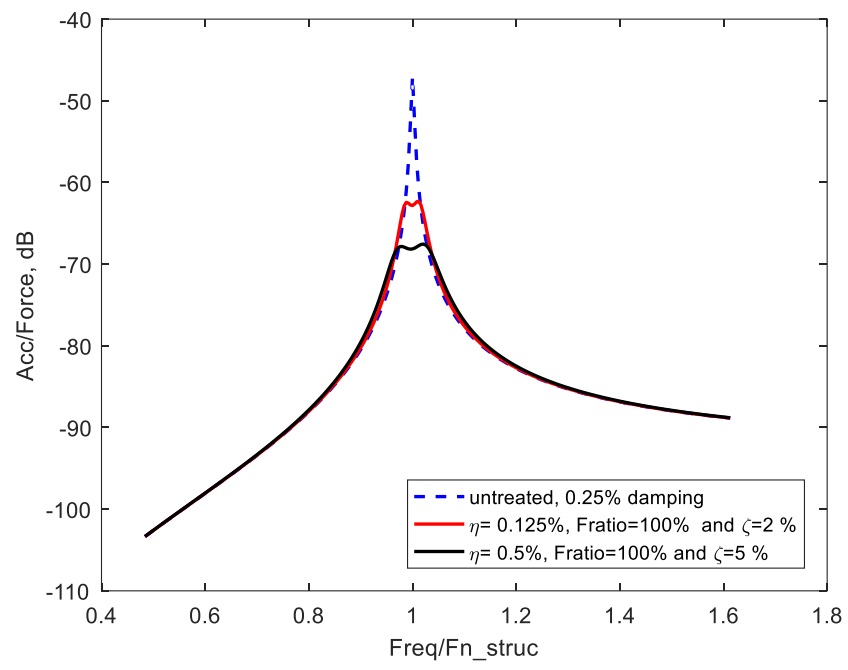

Fig. 1: Frequency response functions of an underdamped structure without and a small (red trace) and large (blue trace) mass ratios .

Clear from Figure 1, increase in mass ratio and the corresponding damping ratio of a TMD increases the energy dissipation (damping) effectiveness as well as the bandwidth of that TMD. As stated earlier, the problem is that in many applications there is neither enough space to accommodate large a large TMD nor the target structure can take the weight of a TMD with large enough mass ratio.

The effectiveness of a tuned mass damper can be increased by introducing a controllable element into its make-up, resulting in an active tuned mass damper (ATMD) [1], [2], [3], [4].

The use of an active tuned mass damper makes it possible to overcome the drawbacks of a passive TMD with small mass ratio. A properly controlled active TMD tuned to a single mode a) has a wider frequency range and thus is more robust (than a passive TMD of the same size) to tuning inaccuracies, b) can self-tune itself, and c) possesses higher effectiveness in adding damping to its target mode. Moreover, an ATMD can be used to add damping to more than one mode of vibration when multiple modes are in need of being damped. This eliminates the need for using multiple passive TMDs with different dynamic characteristics, each tuned to one of the target modes.

Considering that a properly controlled active TMD is a) by far more effective than an equally sized passive TMD and b) can be tuned (add damping) to more than one mode of the structure, it could be an economically attractive solution in certain applications. With cost comparison based on effectiveness, an ATMD would be no more expensive than either a passive TMD of many times larger in size or multiple passive TMDs tuned to multiple frequencies. As to the running cost, with proper design and control strategy an ATMD can switch to a passive system under typical loading conditions, consuming no energy and revert back to active only when the structure reaches a high enough vibration level which requires more effectiveness. Also in the unlikely event of losing power, such active TMD reverts to a passive TMD, automatically.

In this work, a novel APTMD is presented which

1.with a smaller mass possesses the bandwidth and energy dissipation effectiveness of a more massive passive PTMD.

2.It has multiple, adjustable tuning frequencies (and the corresponding internal damping) in multiple directions. With its small size and its multi-frequency tuning capacity, the proposed APTMD is as effective as a passive PTMD many times more massive.

3. Moreover, it can add damping to more than one mode in more than one direction, replacing multiple more massive PTMDs.

As stated earlier, the above-listed attributes lower the cost, weight, and space requirement associated with dampening multiple modes using multiple PTMDs.

Following a short review of existing active tuned damping techniques, the paper introduces the proposed active pendulum tuned mass damper along with its corresponding control strategy. In subsequent sections, the paper continues 
with numerical demonstration of the enhanced damping effectiveness as well as multi-mode damping capacity of the proposed APTMD by incorporating it into the numerical model of a high-rise building perturbed in multiple directions.

\section{Review of ATMD Control Schemes}

Over the last two decades, a number of researchers have suggested different control strategies, mostly for one degree of freedom tuned mass dampers.

Chang and Soong [5], and Isao [5] calculated the required active force using an optimization process and showed significant improvement in the damping effectiveness of the ATMD without increasing its excursion, compared to a passive TMD of equal size. Abdel-Rahman [7] used pole assignment method to evaluate the active force. In separate studies, Chang and Yang [8] and Seshasayee and Yang (1996) computed the active force by feeding back the acceleration, velocity, and displacement of the structure.

In addition to the use of classical feedback control (feeding back displacement, velocity, and acceleration of the structure), modern control technique including linear quadratic regulator (LQR) Alavinasab and Moharrami [5], linear quadratic Gaussian (LQG), $\mathrm{H}_{2}$ and $\mathrm{H}_{\mathrm{inf}}$ control as well as nonlinear control techniques such as sliding-mode control Adhikari and Yamaguchi [9], fuzzy logic Samali et. al. [10], and even neural network control Ghaboussi and Joghataie [11] have been suggested to obtain the most ideal control force for mitigating the vibration of the structure. Despite the successful application of all of these control schemes to a one degree-of-freedom structure, most of these controllers are a) either model-based and require a reasonably accurate model of the structure plus TMD which is rarely available especially in tuned damping applications of very large structures, b) are in need to feeding back all the states of structure and TMD, and/or c) are somewhat complex to implement. These observations and control classifications have been made by Nerves and Krishnan [12] based on the results obtained from the simulation study of applying various active control schemes to large structures.

As will be described in the subsequent sections, the authors have expanded the non-model-based classical feedback control proposed by Nishimura et al. [5] to a multi-degree-of-freedom APTMD and cascaded it with a novel multifrequency tuning strategy.

\section{The Proposed Active Pendulum Tuned Mass Damper (APTMD)}

Figure 2 depicts the proposed APTMD with the mass suspended by steel wire ropes and actuated by 6 hydraulic cylinders (actuators). The hydraulic cylinders along with the moving mass form a 6-legged closed-chain mechanism with 6 degrees of freedom. This mechanism, commonly known as Stewart platform, is capable of moving in any direction and orientation, generating controllable dynamic motions [6] [7] [8]. The hydraulic cylinders (legs) are the active elements of the APTMD realizing the spatial motion of the pendulum mass, decided by the control algorithm.

Except for the use of hydraulic cylinders in place of passive viscous dampers, the proposed APTMD resembles a passive PTMD commonly used in many modern high-rise buildings and towers. In its default state, the hydraulic cylinders are configured to act as passive viscous dampers turning the device to a passive PTMD. The length of the suspension steel wire ropes are selected such that the default passive PTMD is tuned to the lowest target frequency. When the need arises (depending on the extent and frequency content of the structural vibration) the hydraulic cylinders switch to active actuators and turn the device into an active PTMD with enhanced damping effectiveness as well as multi degree-offreedom tuning capacity (capability).

The hydraulic actuation system of the proposed passive/on-demand active PTMD consists of six double acting single ended cylinders. Each hydraulic cylinder is equipped with a temperature-compensated flow control valve and a 4-way electrohydraulic servo-valve; the former is used when the device is in its passive state and the latter along with the hydraulic power supply are used when the device is in active state. Refer to Eltaeb [4] for more on the hydraulic circuit of the APTMD. 


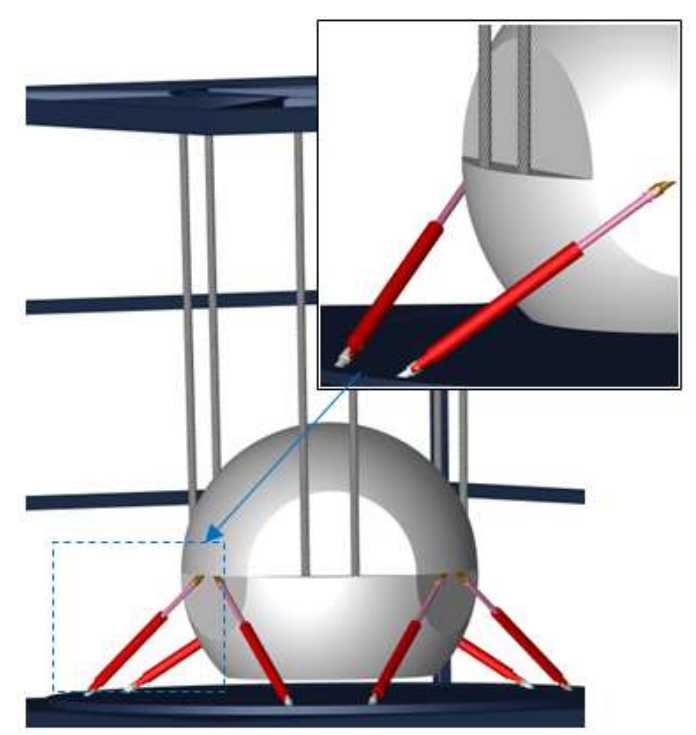

Fig. 2: The active PTMD.

\section{Active Control Strategy}

The control scheme proposed for the multi-degree-of-freedom PTMD is made up of a parallel cascade of a) 'active damping effectiveness enhancement' controller and b) 'active multi-frequency tuning' controller. In addition, a high-level supervisory controller oversees the vibration attributes of the structure both in terms of severity as well as frequency and decides to bring none, one or both controller(s) online.

\subsection{Active Damping Effectiveness Enhancement}

Considering that the inertia force generated by TMD mass is what adds damping to the structure, increasing such inertia force by active means will result in increase in the effectiveness of the TMD. As suggested by Nishimura et. al. [3], the inertia force of a one degree of freedom ATMD appended to a structure can be increased by having the active element actuate the system proportional to the acceleration of the structure. The three degree-of-freedom version of such control strategy formulated in the global Cartesian coordinate system is shown in Equation (1).

$$
U_{2}^{\prime}=\left(\begin{array}{ccc}
G_{X} & 0 & 0 \\
0 & G_{Y} & 0 \\
0 & 0 & G_{\gamma}
\end{array}\right)\left(\begin{array}{l}
\ddot{X}_{s} \\
\ddot{Y}_{s} \\
\ddot{\gamma}_{s}
\end{array}\right)
$$

where $U_{2}^{\prime}$ is the vector of ATMD's active force, $\ddot{X}_{s}, \ddot{Y}_{s}$, and $\ddot{\gamma}_{s}$ are the absolute accelerations of the structure along $\mathrm{x}$ and $\mathrm{y}$ and around $\mathrm{z}$ axes, and $G_{X}, G_{Y}$, and $G_{\gamma}$ are the corresponding feedback gains.

Since the hydraulic cylinders (legs) are to actuate the APTMD, the control force vector which is formulated in the Cartesian coordinate system need to be transformed to the leg space prior to realization.

The control force vector $U_{2}^{\prime}$ of Equation (1) is transformed from the global coordinate system to the legs' coordinate system (leg space) using the principle of virtual work, Eltaeb [4]. Assuming that the friction forces in the joints and the gravitational effects of the legs are negligible and considering that the main gravitational effect due to the weight of the TMD mass is taken up by the steel wire ropes (not the legs of the mechanism), the virtual work contributed by all the active forces is

$$
u_{2}^{\prime T} \cdot \delta p-U_{2}^{\prime T} \cdot \delta P=0
$$


where $\delta p$ and $\delta P$ are the virtual displacements in the local leg space and the global Cartesian coordinate system installed on the structure, respectively. Moreover, $u_{2}^{\prime}$ is the active force vector (realized by hydraulic cylinders) corresponding to $U_{2}{ }^{\prime}$, but defined in the leg space. Clear from Equation (2), the principle of virtual work indicates that the work done by the global force vector $U_{2}^{\prime}$ is the same as the work done by the local force vector $u_{2}^{\prime}$ defined in the leg space.

Using the Jacobian matrix of the closed-chain mechanism $J$, relating small motion of the TMD mass $\delta P$ to the corresponding small motion of the legs $\delta p$, i.e.,

$$
\delta p=J \cdot \delta P
$$

and substituting $\delta p$ in Equation (2) from Equation (3) results in Equation (4)

$$
\left(u_{2}^{\prime T} J-U_{2}^{\prime T}\right) \delta P=0
$$

Considering that the above equation holds for any virtual displacement, one can conclude that

$$
u_{2}^{\prime T} \cdot J-U_{2}^{\prime T}=0 \rightarrow u_{2}^{\prime}=J^{-T} \cdot U_{2}^{\prime}
$$

The actuation force vector of Equation (5) could increase the excursion of the APTMD. As suggested by Nishimura et. al. [3] such excursion can be lowered by augmenting the actuation with feeding back the excursion velocity, making the actuator to act as an active viscous damper, as well. It should be noted that the active scheme of Equation (5) with or without velocity feedback can be turned on and off, depending on the frequency and severity of the structural vibration.

\subsection{Active Multi-Frequency Tuning}

To tune the APTMD to different frequencies in different directions and add the desired internal damping to the APTMD corresponding to those tuning frequencies, the APTMDs' frequency/direction dependent stiffness and damping coefficient matrices need to be realized actively by the actuators. This is achieved by active, simultaneous actuation of the actuators (hydraulic cylinders) subjecting the APTMD mass as well as the structure to the combined stiffness plus damping force vector of $U_{2}^{\prime \prime}$ shown in Equation (6)

$$
U_{2}^{\prime \prime}=K \cdot P+C \cdot \dot{P}=\left(\begin{array}{ccc}
K_{X} & 0 & 0 \\
0 & K_{Y} & 0 \\
0 & 0 & K_{\gamma}
\end{array}\right)\left(\begin{array}{l}
X \\
Y \\
\gamma
\end{array}\right)+\left(\begin{array}{ccc}
C_{X} & 0 & 0 \\
0 & C_{Y} & 0 \\
0 & 0 & C_{\gamma}
\end{array}\right)\left(\begin{array}{l}
\dot{X} \\
\dot{Y} \\
\dot{\gamma}
\end{array}\right)
$$

where $K_{i}$ and $C_{i}$ are the additional stiffness and damping coefficients needed in $i=X, Y$, and $\gamma$ directions, over and beyond what the pendulum itself is providing. And $P=\left[\begin{array}{lll}X & Y & \gamma\end{array}\right]^{T}$ and $\dot{P}=\left[\begin{array}{lll}\dot{X} & \dot{Y} & \dot{\gamma}\end{array}\right]^{T}$ are the position and velocity vectors of the center of mass of the PTMD mass measured in the Cartesian coordinate system installed on the structure, i.e. the relative motion of the APTMD mass with respect to the structure. Note that the formulation of Equation (6) is based on the assumption of the three target modes of the structure are nearly decoupled. If the natural modes were not nearly decoupled then the desired stiffness and damping coefficient matrices shown in Equation (6) would not be symmetric. 
Considering the inconvenience of measuring $\left[\begin{array}{lll}X & Y & \gamma\end{array}\right]^{T}$ and yet the ease of measuring the displacement of the actuators (legs of the Stewart platform) the control force $U_{2}{ }^{\prime \prime}$ of Equation (6) is transformed from the Cartesian coordinate system installed on the structure to the control force defined in the local coordinate systems of the legs. This results in the leg space control force vector $u_{2}$ shown by Equation (7)

$$
u_{2}^{\prime \prime}=k \cdot p+c \cdot \dot{p}
$$

where $p$ and $\dot{p}$ are the displacement and the velocity vectors of the legs, $k$ and $c$ are the stiffness and damping coefficient matrices of the legs defined by Equations (8) and (9)

$$
\begin{aligned}
& k=J^{-T} K J^{-1} \\
& c=J^{-T} C J^{-1}
\end{aligned}
$$

and superscript - $T$ signifies the 'inverse of transpose'; see Eltaeb [4].

As stated earlier the supervisory controller continuously assesses the vibration conditions of the structure, both in terms of severity as well as frequency, decides to activate none, one (either $u_{2}^{\prime}$ or $u_{2}{ }^{\prime \prime}$ ), or both of the two control schemes discussed above.

The block diagram presentation of the structure and the passive/on-demand active PTMD is shown in Figure 3.

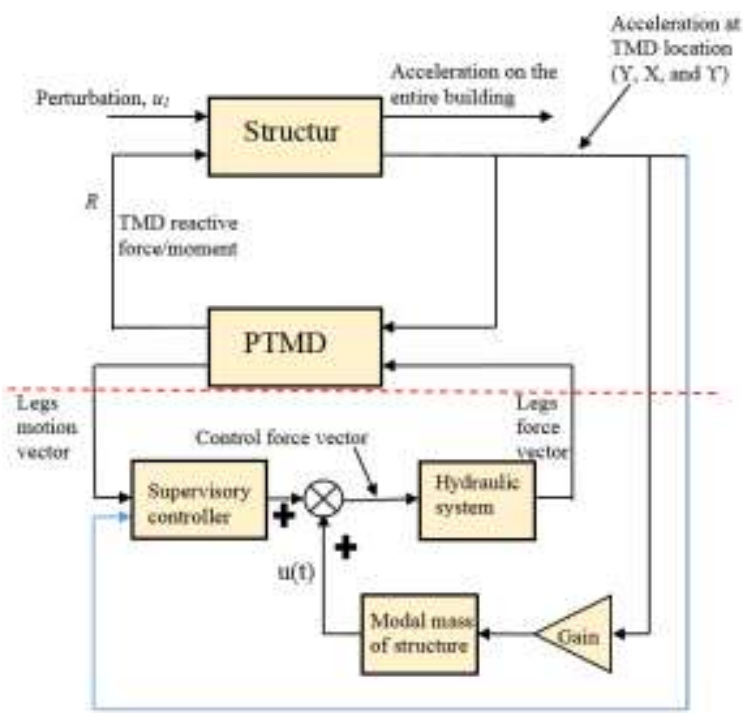

Fig. 3: Block diagram of the structure + APTMD.

\section{Illustrative Example}

The enhanced effectiveness and multi-frequency tuning capability of the proposed APTMD are numerically demonstrated by introducing it into the model of a multi-degree of freedom asymmetrical high-rise building. The building has 41 stories with each floor having three degrees of freedom, two translational in $\mathrm{X}$ and $\mathrm{Y}$ directions and one rotational, denoted by $\gamma$, around the $\mathrm{Z}$ axis. Due to the uniformity in geometry and mass distribution in each floor, vibration in those three directions is nearly decoupled from each other.

The numerical model of the building, in which the first 15 modes of vibration are included, is developed. The nearly decoupled modes of vibration of the building allows for associating each mode with the vibration in one direction, only. 
The proposed APTMD with its six hydraulic cylinders (actuators) is synthesized to add damping to the first three nearly-decoupled modes of the aforementioned tall building with the natural frequencies of $0.18,0.29,0.56 \mathrm{~Hz}$, simultaneously. Figures $4(\mathrm{a}, \mathrm{b}$, and $\mathrm{c})$ show the frequency response functions as well as the resonant time traces of the structure's acceleration along the $\mathrm{Y}\left(1^{\text {st }}\right.$ mode $), \mathrm{X}\left(2^{\text {nd }}\right.$ mode $)$, and $\gamma\left(3^{\text {rd }}\right.$ mode $)$ directions measured at the top floor, without and with the APTMD. Harmonic perturbation with spatially varying amplitude along the height of the structure is used to perturb all the floors, in three directions, simultaneously.

The effectiveness of the APTMD is increased (beyond that of a passive PTMD of the same size) by feeding back the top floor's accelerations measured in the three directions of $\mathrm{X}, \mathrm{Y}$, and $\gamma$ to the controller of Equation (1). In addition to being able to replace multiple passive PTMDs each tuned to a different frequency (in applications where more than one mode are in need of damping), the proposed APTMD performs as robustly and as effectively as a passive PTMD twice as massive.

\section{Summary}

A novel passive pendulum tuned mass damper (PTMD) which on-demand can switch to an active PTMD is proposed. In its default state, the proposed device is passively tuned to the first mode of the structure and acts as a traditional passive PTMD. In its active mode, the parallel cascade of two control schemes a) increases the damping effectiveness of a small APTMD to the same level as a larger passive PTMD tuned to the same frequency and b) tunes the APTMD to multiple frequencies.

With its small size and multi-frequency tuning capacity, the proposed APTMD is as effective as a passive PTMD twice as massive. Moreover, it adds damping to more than one mode in more than one direction, replacing multiple more massive PTMDs each tuned to a different frequency. The enhanced effectiveness and multi-frequency tuning capacity of the proposed APTMD are numerically demonstrated by introducing it into the model of a multi degree-of-freedom asymmetrical, 41 story high-rise building perturbed by wind in multiple directions.
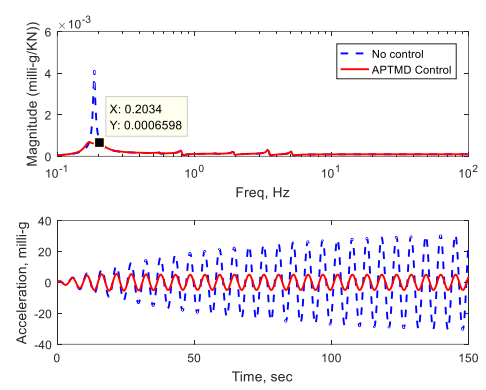

(a)
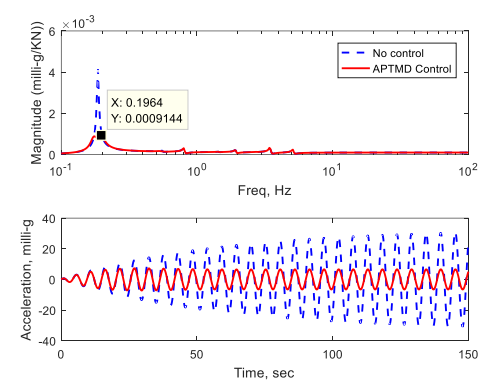

(b)
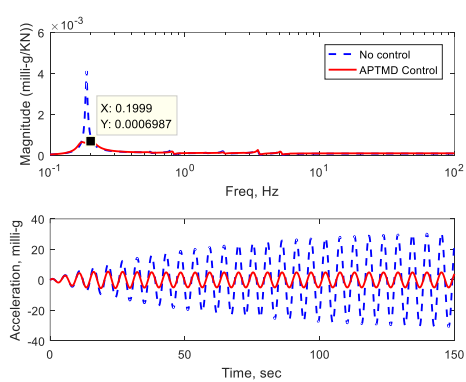

(c)

Fig. 4: FRFs, and the resonant time traces of the structure's linear acceleration along (a) Y, (b) X, and (c) directions measured at the top floor.

\section{References}

[1] J. C. Chang, T. T. Soong, "Structural control using active tuned mass dampers," Journal of the Engineering Mechanics Division, vol. 106, no. 6, pp. 1091-8, 1980.

[2] K. C Kwok, B. Samali, "Performance of tuned mass dampers under wind loads," Engineering Structures., vol. 17, no. 9, pp. 655-67, 1995.

[3] I. Nishimura, T. Kobori, M. Sakamoto, N. Koshika, K. Sasaki, S. Ohrui, “Active tuned mass damper," Smart Materials and Structures, vol. 1, no. 4, p. 306, 1992.

[4] M. A. Eltaeb, "Active Control of Pendulum Tuned Mass Dampers for Tall Buildings Subject to Wind Loads," Ph.D. dissertation, The University of Dayton, 2017.

[5] A. Alavinasab, H. Moharrami, A. Khajepour "Active Control of Structures Using Energy-Based LQR Method," Computer-Aided Civil and Infrastructure Engineering, vol. 21, no. 8, pp. 605-11, 2006. 
[6] B. Samali, M. Al-Dawod, K. C. Kwok, F. Naghdy, "Active control of cross wind response of 76-story tall building using a fuzzy controller," Journal of engineering mechanics., vol. 130, no. 4, pp. 492-8, 2004.

[7] J. Ghaboussi, A. Joghataie, "Active control of structures using neural networks," Journal of Engineering Mechanics., vol. 121, no. 4, pp. 555-67, 1995.

[8] R. Adhikari, H. Yamaguchi, "Sliding mode control of buildings with ATMD," Earthquake engineering \& structural dynamics., vol. 26, no. 4, pp. 409-22, 1997.

[9] A. Alavinasab, H. Moharrami, A. Khajepour, "Active Control of Structures Using Energy-Based LQR Method," Computer-Aided Civil and Infrastructure Engineering., vol. 21, no. 8, pp. 605-11, 2006. 